\title{
Kooperative Phänomene und Diffusion eines Plasmas quer zu einem Magnetfeld II
}

\author{
Von D. Pfirsch und L. Biermann \\ Aus dem Max-Planck-Institut für Physik und Astrophysik, München \\ (Z. Naturforschg. 15 a, 14-18 [1960]; eingegangen am 15. September 1959)
}

\begin{abstract}
By means of two examples of inhomogeneous plasmas the occurence of microscopic instabilities is investigated. It is shown, that an inhomogeneous one-component plasma with diffusion but without a magnetic field is stable in the limiting case of linearised theory and that in a similar plasma without diffusion, which is confined by a magnetic field, an instability of apparently fairly general character exists, but its range is not yet known.
\end{abstract}

In der vorangehenden Arbeit ${ }^{1}$ wurden allgemeine Gründe angegeben, die das Auftreten von Instabilitäten in mikroskopischen Bereichen bei inhomogenen Plasmen wahrscheinlich erscheinen ließen. Auch die Bedeutung solcher Instabilitäten im Hinblick auf das Festhalten von Plasmen mittels Magnetfelder, d. h. auf die Diffusion von Plasmen quer zu einem Magnetfeld, wurde dort erläutert. Die vorliegende Arbeit beschäftigt sich in mehr expliziter Weise mit der Wellenausbreitung in inhomogenen Plasmen und untersucht in folgenden Fällen deren Stabilitätsverhalten:

Störungen kleiner Amplituden

1. in einem einkomponentigen Plasma ohne Magnetfeld mit Dichtegradienten und Teilchendiffusion,

2. in einem einkomponentigen Plasma, das durch ein Magnetfeld eingeschlossen ist, ohne Diffusion.

Einige Aussagen werden dann noch über einen dritten Fall

3. Störungen kleiner Amplituden in einem einkomponentigen Plasma, das durch ein Magnetfeld eingeschlossen ist, mit Diffusion

gemacht.

Der Theorie liegt das gekoppelte System von Einteilchen-Boltzmans-Gleichung und Maxwellschen Gleichungen zugrunde. Die Lösung der linearisierten Boltzmann-Gleichung erfolgt nach dem Charakteristikenverfahren (siehe Anhang). Es wird sich zeigen, daß in bestimmten Fällen Instabilitäten auftreten, welche die makroskopische Theorie nicht ergibt.

\section{Dichtegradient mit Teilchendiffusion ohne Magnetfeld}

Das ungestörte Plasma wird näherungsweise durch eine Verteilungsfunktion

$$
f(x, \mathfrak{b})=n\left[x-v_{x} \tau(v)\right] f_{0}(v)
$$

beschrieben. Darin ist $f_{0}(v)$ die MaxweLL-Verteilung, $n(x)$ ist im wesentlichen die räumliche Teilchendichte, $\tau(v)$ ist eine Funktion, die von der freien Weglänge der Teilchen abhängt. Sie stellt so etwas wie eine freie Flugzeit dar. In welcher Weise durch (1) eine Diffusion wiedergegeben wird, erhellt, wenn man (1) annähert durch

$$
f(x, \mathfrak{b}) \approx n(x) f_{0}(v)-v_{x} \tau(v) \frac{\mathrm{d} n}{\mathrm{~d} x} f_{0}(v) .
$$

Berechnet man hiermit den Diffusionsstrom, so folgt $j_{x}=\int v_{x} f \mathrm{~d}^{3} \mathfrak{b}=-\frac{\mathrm{d} n}{\mathrm{~d} x} \int v_{x}^{2} \tau(v) f_{0}(v) \mathrm{d}^{3} \mathfrak{b} ;$

der Diffusionsstrom ist also bei langsam veränderlicher Dichte gerade durch den Gradienten von $n(x)$ bestimmt.

Gl. (1) löst genähert die stationäre FoKKeRPlanck-Gleichung

$$
\begin{aligned}
v_{x} \frac{\partial f}{\partial x}=\beta \nabla_{\mathfrak{v}}\left(\frac{\mathfrak{v}}{v^{3}}\right. & \exp \left\{v^{2} / 2 V^{2}\right\} f \\
& \left.+\frac{V^{2}}{v^{3}} \exp \left\{v^{2} / 2 V^{2}\right\} \nabla_{\mathfrak{v}} f\right)
\end{aligned}
$$

$\beta=$ const,$V^{2}=$ const $=$ mittlere quadratische Geschwindigkeit der Teilchen.

Dabei ist die Näherung dadurch bestimmt, daß die freie Weglänge der Teilchen klein gegenüber $n(x) /(\mathrm{d} n / \mathrm{d} x)$ sein soll. Wegen der Abhängigkeit der rechten Seite der Gl. (4) von $v$ ist dies für alle $v$ erfüllbar.

Der Fonker-Planck-Term auf der rechten Seite von (4) ist vom Typ

$$
\nabla_{\mathfrak{v}}\left[\mathfrak{v} g(v) f+h(v) \nabla_{\mathfrak{v}} f\right] .
$$

(5) stellt eine Einschränkung gegenüber dem allgemeinsten kugelsymmetrischen Ansatz dar, die in der Beschränkung auf den Skalar $h(v)$ besteht, neben dem noch ein Tensor $h_{1}(v) v_{i} v_{k}$ möglich wäre. Die

1 L. Biermann u. D. Pfirsch, Z. Naturforschg. 15 a, 10 [1960]; im folgenden als I zitiert. 
Forderung, daß (5) neben dem von selbst erfüllten lokalen Teilchenerhaltungssatz lokal auch den Energiesatz erfüllt und die MaxwelL-Verteilung zur Lösung hat, liefert

$$
\begin{gathered}
h(v)=\frac{a+b v^{2}}{v^{3}} \exp \left\{v^{2} / 2 V^{2}\right\}, \\
g(v)=\frac{1}{v^{3} V^{2}}\left(a+2 b V^{2}+b v^{2}\right) \exp \left\{v^{2} / 2 V^{2}\right\}
\end{gathered}
$$

mit konstanten $a$ und $b . b=0$ führt auf den FokKERPlanck-Term in (4), mit dem man z. B. eine elektrische Leitfähigkeit proportional $T^{3 / 2}$ berechnen kann *.

Um den gewohnten Ausdruck für die elektrische Leitfähigkeit zu bekommen, hat man

$$
\beta=2,5 \frac{n_{0} e^{4}}{m^{2}} \lg \Lambda
$$

zu setzen, wodurch $\beta$ festgelegt wird.

$\tau(v)$ hat die Form

$$
\tau(v)=\varepsilon v^{2}, \quad \varepsilon \approx 0,25 \frac{V}{\beta}
$$

Der Impulssatz wird durch (4) nicht erfüllt. (4) schließt also Stöße ohne Energieaustausch mit einem fremden System ein, wodurch ein quasistationärer Dichtegradient ermöglicht wird.

Das Bild, das Gl. (4) zugrunde liegt, entspricht also etwa dem einer Flüssigkeit, die durch einen Schwamm strömt, oder eines Gases, das durch eine poröse Wand diffundiert.

Für verschwindende freie Weglänge wird $\tau=0$ und Gl. (1) wird eine exakte Lösung, die keine Diffusion mehr enthält. Bei endlicher freier Weglänge beschreibt (1) einen Diffusionsvorgang mit einer durch $\beta$ und $\mathrm{d} n / \mathrm{d} x$ bestimmten Zeitkonstanten. Diese Zeitkonstante kann im Rahmen der Untersuchung immer wesentlich größer als die durch die Wellen im Plasma bestimmten Zeiten angenommen werden, so daß dann bez. der Wellenvorgänge mit der Verteilungsfunktion (1) gerechnet werden kann, wobei auch noch die Stöße in der Gleichung für die Störverteilungsfunktion vernachlässigt werden können.

Die Wellenvorgänge $\sim e^{-i \omega t}$ in einem durch (1) beschriebenen Plasma können noch relativ einfach diskutiert werden für

$$
n(\zeta)=n_{0}\left(1+\frac{\zeta^{2}}{\lambda^{2}}\right), \quad \zeta=x-v_{x} \tau(v) .
$$

Betrachtet man nur longitudinale Wellen, die sich parallel zum Dichtegradienten ausbreiten, dann sind diese Wellen auf ein endliches $x$-Intervall beschränkt; das entspricht gerade den Verhältnissen in einer normalen Plasmaoberfläche, wo Wellen mit $\omega<\omega_{\mathrm{p}_{0}} \quad\left(\omega_{\mathrm{p}_{0}}=\right.$ Plasmafrequenz für das Plasmainnere) nur in einer gewissen Schicht der Oberfläche möglich sind, so daß die mit (7) gewonnenen Ergebnisse als hinreichend allgemein gültig angesehen werden müssen. (7) führt mittels einer räumlichen Fourier-Transformation $\mathfrak{E}(x)=\int \mathfrak{F}(k) e^{i k x} \mathrm{~d} k$ auf eine Differentialgleichung 2. Ordnung im f-Raum für das elektrische Feld F. Die Bedingung, daß F für $\mathfrak{f} \rightarrow \pm \infty$ nicht unendlich groß werden darf, führt auf folgende für Wellenlängen größer als die Debye-Länge (etwa $\lambda>4 \pi \cdot$ Debye-Länge) geltende Dispersionsbeziehung

$$
\omega^{2}=\omega_{\mathrm{p}_{0}}^{2}+\frac{3 k T}{m} k_{0}^{2}\left(1+\frac{5}{6} \frac{\varepsilon^{2}(2 k T / m)^{3}}{\lambda^{2}}\right)-\operatorname{sign} \Re e \cdot \omega \cdot i \frac{5}{2} \frac{\varepsilon(2 k T / m)^{2} \omega_{\mathrm{p}}}{\lambda^{2}}\left(1+13,2 \frac{k T}{m \omega_{\mathrm{p}}^{2}} k_{0}^{2}\right)
$$

bzw. für alle Wellenlängen

$$
\lambda^{2}\left(1-\frac{1}{F_{1} \omega_{\mathrm{p}}^{2}}\right)-\left(\frac{F_{2}}{F_{1}}\right)^{2}+\frac{\mathrm{d}}{\mathrm{d} k_{0}}\left(\frac{F_{2}}{F_{1}}\right)=0 .
$$

Dabei ist $k_{0}$ eine charakteristische Wellenzahl, durch die im wesentlichen eine Art Wellenlänge der Störung festgelegt ist; weiter ist

$$
\begin{gathered}
\omega_{\mathrm{p}_{0}}^{2}=\frac{4 \pi n_{0} e^{2}}{m}, \quad F_{1}(\gamma, \omega)=-i \frac{\pi^{1 / 2}}{2 \gamma}\left\{\frac{\omega}{2 \gamma^{1 / 2}}\left(1+\Phi\left(\frac{i \omega}{2 \gamma^{1 / 2}}\right)\right) \exp \left(-\omega^{2} / 4 \gamma\right)-\frac{i}{\pi^{1 / 2}}\right\}, \\
F_{2}(\gamma, \omega)=-\frac{\varepsilon}{\alpha^{3 / 2}} \frac{\pi^{1 / 2}}{4 \gamma}\left\{\left(-1-\frac{\omega^{2}}{4 \gamma}+\frac{\omega^{4}}{8 \gamma^{2}}\right) \exp \left(-\omega^{2} / 4 \gamma\right)\left(1+\Phi\left(\frac{i \omega}{2 \gamma^{1 / 2}}\right)\right)-\frac{i}{\pi^{1 / 2}} \frac{\omega^{3}}{4 \gamma^{3 / 2}}\right\}, \\
\gamma=k_{0}^{2} / 4 \alpha .
\end{gathered}
$$

(8 a) beschreibt also eine gedämpfte stabile Lösung. Wie eine eingehendere Diskussion zeigt, scheinen instabile Lösungen mit Anwachsraten größer als die Diffusionsrate, d. h. also Anwachsraten, die inner-

* Herr Knorr wies uns freundlicherweise darauf hin, daß $b=0$ wegen des Verhaltens des Stoßterms bei $v=0$ allgemein notwendig ist. 
halb der quasistationären Näherung von Bedeutung sind, nicht zu existieren. Damit liegt die Vermutung nahe, daß diffusionsbedingte Instabilitäten, von denen in I die Rede war, wahrscheinlich nur in nichtlinearen Theorien zu erhalten sind.

\section{Dichtegradient ohne Teilchendiffusion mit Magnetfeld}

Es wurde ein ebenes Plasma mit einem Dichte- gradienten in $x$-Richtung behandelt. Das Magnetfeld liegt in $z$-Richtung. Für $B^{2} / 8 \pi \gg n k T$ kann ein Plasma mit der Dichteverteilung

$$
\begin{aligned}
n(x) & =n_{0} \exp \left\{-\frac{\gamma}{1+\gamma} \frac{x^{2}}{r_{\mathrm{g}}^{2}}\right\}, \quad r_{\mathrm{g}}=\frac{\sqrt{2 m k T}}{e B_{0}} \\
& =\text { mittlerer Gyrationsradius }
\end{aligned}
$$

mit $\gamma=$ const und im allgemeinen klein gegen 1 durch die Verteilungsfunktion

$$
f(x, \mathfrak{b})=\left(\frac{\alpha}{\pi}\right)^{3 / 2}(1+\gamma)^{1 / 2} n_{0} \exp \left\{-\alpha\left(v^{2}+\gamma\left(v_{y}+\omega_{\mathrm{c}} x\right)^{2}\right)\right\}, \alpha=m / 2 k T, \quad \omega_{\mathrm{c}}=\frac{e B_{0}}{m c}
$$

als Lösung der Boltzmann-Gleichung beschrieben werden. $B_{0}$ ist das Magnetfeld für $x \rightarrow \infty$. Aus (11) folgt für das Magnetfeld

$$
B(x)=B_{0}\left(1-\frac{4 \pi n_{0} k T}{B_{0}{ }^{2}} \exp \left\{-\frac{\gamma}{1+\gamma} \frac{x^{2}}{r^{2}}\right\}\right), \quad \frac{1}{8 \pi}\left(B^{2}(\infty)-B^{2}(0)\right) \approx n_{0} k T .
$$

In einem durch (11) beschriebenen Plasma wurden Störungen folgender Symmetrie diskutiert, wobei in der Gleichung für die Störverteilungsfunktion $B(x)$ durch $B_{0}$ ersetzt wurde. $\left[B_{0}\right.$ ist sicher stabiler als $B(x)$, so daß diese Ersetzung eher zu stabilen als zu instabilen Lösungen führen sollte.]
(a)
$\mathfrak{f}=\left(k_{x}, 0,0\right)$
$\mathfrak{E}=\left(0,0, E_{z}\right)$,
(b)$$
\mathfrak{l}=\left(k_{x}, 0,0\right), \quad \mathfrak{F}=\left(E_{x}, E_{y}, 0\right) .
$$

Im Fall $T=0$ ergibt sich für $E_{z}$ [Fall (a)] bzw. $E_{y}[$ Fall (b) ] die - aus der makroskopischen Theorie schon bekannte - Gleichung

$$
E . .^{\prime \prime}+\frac{\omega^{2}}{c^{2}} n_{\mathrm{R}}(x) E .=0 .
$$

Hier ist $n_{\mathrm{R}}(x)$ der ortsabhängige Brechungsindex, der aus dem bekannten ortsunabhängigen Brechungsindex durch die Ersetzung von $\omega_{\mathrm{p}}$ durch $\omega_{\mathrm{p}}(x)$ folgt.

(13) enthält natürlich nur stabile Lösungen, da für $T=0$ gar keine Energie verfügbar ist, mittels der sich Instabilitäten entwickeln könnten.

Neben (13) erhält man im Fall (b) noch eine Beziehung, die $E_{x}$ aus $E_{y}$ zu berechnen erlaubt:

$$
E_{x}=i \frac{\omega_{\mathrm{c}}}{\omega} \frac{\omega_{\mathrm{p}}^{2}(x)}{\omega^{2}-\omega_{\mathrm{c}}{ }^{2}-\omega_{\mathrm{p}}^{2}(x)} E_{y}(x) .
$$

Außerhalb des Plasmas ist $\omega_{\mathrm{p}}(x)=0$, dort verschwindet also $E_{x}$ entsprechend der Tatsache, daß im Vakuum keine longitudinalen Wellen möglich sind.

Für das mögliche Auftreten von Instabilitäten ist nur der Fall $T \neq 0$ interessant. Für (a) gilt hier ähnliches wie im Fall $T=0$, die rein transversale
Welle ist also auch hier stabil. Die Wellen der durch (b) beschriebenen Art haben im allgemeinen longitudinale und transversale Komponenten des elektrischen Feldes, es gibt von ihnen zwei Typen, von denen der eine den rein transversalen, der andere den rein longitudinalen Wellen für verschwindendes äußeres Magnetfeld entspricht.

Für kleinere Temperaturen $\left(\frac{1}{4} k^{2} r_{\mathrm{g}}^{2} \sin ^{2}\left|\frac{\omega_{\mathrm{c}}}{\omega}\right| \ll 1\right)$ erhält man für sie weit außerhalb des Plasmas $\left(\gamma \frac{x^{2}}{r^{2}} \gg 1\right)$ folgende Gleichungen 4. Ordnung für $E_{y}$

$$
\begin{gathered}
E_{y}{ }^{(4)} \approx-\frac{1}{A} \frac{\omega^{2}}{c^{2}}\left(E_{y}{ }^{(2)}+\frac{\omega^{2}}{c^{2}} E_{y}\right) \exp \gamma \frac{x^{2}}{r_{\mathrm{g}}{ }^{2}}, \\
A=\frac{3}{2}(1+\gamma)^{1 / 2} \frac{1}{\alpha c^{2}} \frac{\omega^{2} \omega_{\mathrm{p}_{0}}{ }^{2}}{\left(\omega^{2}-\omega_{\mathrm{c}}{ }^{2}\right)\left(\omega^{2}-4 \omega_{\mathrm{c}}{ }^{2}\right)} .
\end{gathered}
$$

Diese Gleichung hat die angegebenen zwei wesentlich verschiedenen Lösungspaare. Die beiden Lösungstypen sind

$$
\begin{aligned}
& \left|E_{y}{ }^{(4)}\right| \\
& \ll \mid \frac{1}{A} \frac{\omega^{2}}{c^{2}} \exp \left\{\gamma \frac{x^{2}}{r_{\mathrm{g}}{ }^{2}}\right\}\left(\left|E_{y}{ }^{(2)}\right|+\frac{\omega^{2}}{c^{2}}\left|E_{y}\right|\right)
\end{aligned}
$$$$
\text { also } E_{y}^{(2)}+\frac{\omega^{2}}{c^{2}} E_{y} \approx 0, \quad E_{y} \sim \exp \left\{ \pm i \frac{\omega}{c} x\right\}
$$

$$
\left|\frac{\omega^{2}}{c^{2}} E_{y}\right| \ll\left|E_{y}^{(2)}\right| \text {, }
$$

also $\quad E^{(4)}+\frac{1}{A} \frac{\omega^{2}}{c^{2}} \exp \left\{\gamma \frac{x^{2}}{r_{\mathrm{g}}^{2}}\right\} E_{y}^{(2)} \approx 0$.

Die Wellen vom Typ (I) gehen außerhalb des Plasmas in normale transversale ebene elektromagneti- 
sche Wellen über; mit diesem Wellentyp hat man z. B. bei Mikrowellenuntersuchungen zu tun. Er ist dadurch charakterisiert, daß er für $T=0$ einen endlichen Brechungsindex besitzt. Diese Wellen kommen auch schon in der makroskopischen Theorie vor, nicht dagegen die Wellen vom Typ (II), durch die eine Bewegungsmöglichkeit des Plasmas erfaßt wird, für die erst die mikroskopische Theorie eine Beschreibung zuläßt.

Diese Wellen bleiben nur für $A / \omega^{2}>0$ im unendlichen endlich. In diesem Fall sind sie dann auch im Plasma eingeschlossen: Mittels der WBK-Methode erhält man eine genäherte Lösung von der Form

$$
\begin{aligned}
& E_{y}^{(2)} \sim \exp \left\{-\frac{1}{4} \gamma \frac{x^{2}}{{r \mathrm{~g}^{2}}^{2}}\right\} \cdot \exp \left\{ \pm i\left(\frac{2}{3(1+\gamma)^{1 / 2}} \alpha \frac{\left(\omega^{2}-\omega_{\mathrm{c}}^{2}\right)\left(\omega^{2}-4 \omega_{\mathrm{c}}^{2}\right)}{\omega_{\mathrm{p}_{0}}{ }^{2}}\right)^{1 / 2}\right\} \cdot I, \\
& \text { wo } I=\int^{x} \exp \left\{\frac{1}{2} \gamma \frac{\xi^{2}}{r^{2}} \mathrm{~d} \xi\right\} \text {, }
\end{aligned}
$$

die dies deutlich zeigt. Darin verhält sich $I$ für große $x$ wie

$$
r_{\mathrm{g}} \frac{1+\gamma}{\gamma} \frac{r_{\mathrm{g}}}{x} \exp \left\{\frac{1}{2} \gamma \frac{x^{2}}{r_{\mathrm{g}}^{2}}\right\} .
$$

Die auf Grund der Grenzbedingungen zugelassenen Frequenzen sind bestimmt durch

$$
\left(\omega^{2}-\omega_{\mathrm{c}}^{2}\right)\left(\omega^{2}-4 \omega_{\mathrm{c}}^{2}\right)=\Omega^{4}>0
$$

eder

$$
\omega^{2}=\frac{5}{2} \omega_{\mathrm{c}}^{2} \pm \sqrt{\Omega^{4}+\frac{99}{4} \omega_{\mathrm{c}}^{4}} .
$$

Es gibt also einen verbotenen Bereich für $\omega$, der zwischen der einfachen und der doppelten Gyrationsfrequenz liegt. Das wesentliche ist aber, daß $\omega^{2}$ jeden negativen Wert annehmen kann. Dazu muß nur $|\Omega|>\sqrt{2}\left|\omega_{\mathrm{c}}\right|$ sein. Die Konfiguration ist also instabil. Die Instabilitäten besitzen gemäß (16) eine durch $|\Omega|>\sqrt{2}\left|\omega_{\mathrm{c}}\right|$ bestimmte maximale lokale Wellenlänge

$$
\begin{aligned}
& \frac{\frac{\lambda}{2 \pi}<r_{\mathrm{g}} \frac{c}{v_{\mathrm{A}}}\left(\frac{3}{8}\right)^{1 / 2}(1+\gamma)^{1 / 4},}{\left(4 \pi n_{0} \exp \left\{-\gamma \frac{x^{2}}{v_{\mathrm{g}}^{2}}\right\} m c^{2}\right)^{1 / 2}} \\
& \quad=\text { ALFVÉN-Geschwindigkeit am Ort } x .
\end{aligned}
$$

Die Instabilität wird durch den Gyrationsradius, also durch eine mikroskopische Größe bestimmt, sie kann daher nicht aus der üblichen makroskopischen Theorie folgen; das entspricht wieder der Tatsache, $\mathrm{da} ß$ dieser Lösungstyp in der makroskopischen Theorie gar nicht auftritt. Da außerdem der Gyrationsradius für $T=0$ verschwindet, existiert auch diese Instabilität nicht für $T=0$ in Übereinstimmung mit allgemeinen Überlegungen. Der Gültig- keitsbereich für diese Rechnung ist entsprechend dem Vorangehenden gegeben durch die Relationen $k^{2}{r_{\mathrm{g}}}^{2} \ll 1, \quad \frac{1}{A} \exp \left\{\gamma x^{2} / r_{\mathrm{g}}{ }^{2}\right\} \gg 1, \quad \frac{B^{2}}{8 \pi} \gg n k T$.

Durch Einsetzen von $\lambda / 2 \pi=1 / k$ aus (19) und A aus (15) folgt hieraus der Reihe nach

$$
\begin{aligned}
& \frac{\omega_{\mathrm{c}}^{2}}{\omega_{\mathrm{p}_{0}}{ }^{2}} \exp \left\{\gamma x^{2} / r_{\mathrm{g}}^{2}\right\} \ll 1, \\
& \alpha c^{2} \frac{\omega_{\mathrm{c}}{ }^{2}}{\omega_{\mathrm{p}_{0}}{ }^{2}} \exp \left\{\gamma x^{2} / \mathrm{r}_{\mathrm{g}}{ }^{2}\right\} \gg 1, \quad \alpha c^{2} \frac{\omega_{\mathrm{c}}{ }^{2}}{\omega_{\mathrm{P}_{0}}{ }^{2}} \gg 1 \text {. }
\end{aligned}
$$

Diese Bedingungen können also gemeinsam erfüllt werden für hinreichend kleines $\omega_{\mathrm{c}}$ im Vergleich zu $\omega_{\mathrm{p} 0}$ und für hinreichend großes $\alpha c^{2}$ im Vergleich zu 1 , also für genügend kleine Temperaturen. Wir vermuten, daß die von uns gefundenen Instabilitäten allgemeiner, als diesen Bedingungen entspricht, auftreten werden, doch konnte das bis jetzt noch nicht genauer untersucht werden.

Was den Einfluß dieser Instabilitäten auf die Diffusion entsprechend den Formeln (3) und (6) in I betrifft, sei bemerkt, daß wir zwar keine Überstabilitäten gefunden haben, so daß nicht ohne weiteres klar ist, wie (3) angewendet werden soll. Soweit aber der Gyrationsradius der Ionen wesentlich gröBer als die Wellenlänge der instabilen Wellen ist, also größer als der Gyrationsradius der Elektronen, machen sich diese Instabilitäten gleich Überstabilitäten mit einer Frequenz

$$
v=k v_{\mathrm{i}}
$$

bemerkbar, wenn $v_{\mathrm{i}}$ die thermische Geschwindigkeit der Ionen ist. 


\section{Dichtegradient mit Teilchendiffusion und mit Magnetfeld}

Hier können erst Vermutungen auf Grund der vorangehenden Rechnungen ausgesprochen werden. Danach wäre zu erwarten, daß Dämpfung wie bei 1) und Instabilitäten wie bei 2) auftreten, die sich also überlagern werden, und daß es der Lösungstyp II des vorigen Abschnittes ist, der diese Eigenschaften zeigt. Detaillierte Rechnungen sind im Gange.

Den Herren Dr. v. Hagenow und Dr. Hain möchten wir für zahlreiche Diskussionen herzlich danken.

\section{Anhang:}

\section{Lösung der linearisierten Boltzmann-Gleichung}

Die linearisierte Boltzmans-Gleichung hat in allen Beispielen die Form

$$
\frac{\partial f_{1}}{\partial t}+\mathfrak{v} \nabla_{\mathfrak{r}} f_{1}+\frac{1}{m} \mathfrak{R}_{0}(\mathfrak{r}, \mathfrak{v}) \nabla_{\mathfrak{v}} f_{1}=-\frac{1}{m} \Re_{1}(\mathfrak{r}, \mathfrak{v}, t) \nabla_{\mathfrak{v}} f_{0} .
$$

Darin sind $f_{0}$ die ungestörte Verteilungsfunktion und $\Omega_{0}(\mathfrak{r}, \mathfrak{v})$ die ungestörte Kraft, $f_{1}$ ist die Störung der Verteilungsfunktion, $\Re_{1}$ die Störung der Kraft. Zu (A. 1) gehören die Gleichungen für die Charakteristiken, die eine Konstruktion der Lösung mittels Integrationskonstanten ermöglichen:

$$
\frac{\mathrm{d} \mathfrak{r}}{\mathrm{d} t}=\mathfrak{v}, \quad \frac{\mathrm{d} \mathfrak{v}}{\mathrm{d} t}=\frac{1}{m} \Re_{0}(\mathrm{r}, \mathfrak{v}), \quad \frac{\mathrm{d} f_{1}}{\mathrm{~d} t}=-\frac{1}{m} \Re_{1}(\mathfrak{r}, \mathfrak{v}, t) \nabla \mathfrak{v} f_{0},
$$

deren Lösungen sind

$$
\mathfrak{r}(t)=\mathfrak{r}\left(\mathfrak{r}^{0}, \mathfrak{v}^{0}, t\right), \quad \mathfrak{v}(t)=\mathfrak{v}\left(\mathrm{r}^{0}, \mathfrak{v}^{0}, t\right), \quad f_{1}(t)=f_{1}{ }^{0}-\frac{1}{m} \int_{0}^{t} \mathfrak{K}_{1}[\mathrm{r}(\tau), \mathfrak{v}(\tau), \tau] \cdot \nabla_{\mathfrak{v}} f_{0}[\mathfrak{r}(\tau), \mathfrak{v}(\tau)] \mathrm{d} \tau .
$$

Die oben mit Null indizierten Größen stellen Anfangswerte, also Integrationskonstanten dar. Man kann (A. 3) nach diesen Integrationskonstanten auflösen und erhält

$$
\begin{gathered}
\quad \mathfrak{r}^{0}=\mathfrak{r}^{0}[\mathfrak{r}(t), \mathfrak{v}(t) t], \quad \mathfrak{v}^{0}=\mathfrak{v}^{0}[\mathfrak{r}(t), \mathfrak{v}(t) t], \\
f_{1}{ }^{0}=f_{1}(t)+\frac{1}{m} \int_{0}^{t} \Re_{1}\left(\mathfrak{r}\left(\mathfrak{r}^{0}[\mathfrak{r}(t), \mathfrak{v}(t), t], \mathfrak{v}^{0}(\ldots) \tau\right), \mathfrak{v}(\ldots), \tau\right) \nabla_{\mathfrak{v}} f_{0}[\mathfrak{r}(\ldots), \mathfrak{v}(\ldots)] \mathrm{d} \tau,
\end{gathered}
$$

wobei die Klammern mit den Punkten immer entsprechende Argumente wie bei $\mathfrak{r}$ und $\mathfrak{r}^{0}$ bedeuten sollen. Ersetzt man in (A. 4) $\mathfrak{r}(t), \mathfrak{v}(t), f_{1}(t)$ durch $\mathfrak{r}, \mathfrak{v}, f_{1}$, dann impliziert jede Funktion der Größen (A. 4) gleich Null gesetzt eine Lösung der linearisierten Boltzmann-Gleichung (A. 1). Alle diese Beziehungen können als Anfangsbedingungen für $f_{1}$ gedeutet werden, so daß wir als allgemeine Lösung für $f_{1}$ erhalten

$f_{1}(\mathfrak{r}, \mathfrak{v}, t)=f_{1}{ }^{0}\left[\mathfrak{r}^{0}(\mathfrak{r}, \mathfrak{v}, t), \mathfrak{v}^{0}(\mathfrak{r}, \mathfrak{v}, t)\right]-\frac{1}{m} \int_{0}^{t} \Re_{1}\left(\mathfrak{r}\left(\mathfrak{r}^{0}(\mathfrak{r}, \mathfrak{v}, t) \mathfrak{v}^{0}(\ldots), \tau\right), \mathfrak{v}(\ldots), \tau\right) \nabla_{\mathfrak{v}} f_{0}[\mathfrak{r}(\ldots), \mathfrak{v}(\ldots)] \mathrm{d} \tau$.

Wie eine genauere Diskussion zeigt, kann für hinreichend glatte Anfangsstörungen $f_{1}{ }^{0}$ und ungestörte Verteilun gen $f_{0}$ bei der Berechnung makroskopischer Größen mittels $f_{1}$ für große Zeiten $f_{1}{ }^{0}$ vernachlässigt und die Integrationsgrenze 0 durch $-\infty$ ersetzt werden. Man hat es dann formal mit einer Darstellung von $f_{1}$ zu tun, die schon von Drummond ${ }^{2}$ angegeben wurde, die aber im allgemeinen nur hinsichtlich der Berechnung makroskopischer GröBen, wie Ladungs- und Stromdichten, sinnvoll ist.

2 J. E. Drummond, Phys. Rev. 110, 293 [1958]. 